\section{La keyicine}

\section{Quand deux têtes valent mieux qu'une pour identifier un antibiotique !}

Alex Carignan, Louis-Charles Fortier
Département de microbiologie et d'infectiologie,

Faculté de médecine et des sciences de la santé,

Université de Sherbrooke, $3001,12^{\mathrm{e}}$ avenue Nord, Sherbrooke, JlH 5 N4 Canada.

alex.carignan@usherbrooke.ca
Antibiorésistance et développement de nouveaux antibiotiques

La résistance aux antibiotiques constitue une menace de santé publique grandissante à l'échelle planétaire. L'Organisation mondiale de la santé a récemment publié un plan d'action pour combattre la résistance aux antimicrobiens [1]. Le but de ce plan est de maintenir le plus longtemps possible la capacité de traiter et de prévenir les maladies infectieuses au moyen de médicaments sûrs et efficaces, utilisés de manière responsable, et accessibles à tous ceux qui en ont besoin.

Ces recommandations s'effectuent dans un contexte où le développement de nouveaux antibiotiques à l'aide de cultures microbiennes conventionnelles est de plus en plus limité [2]. Les avancées dans les domaines de la génomique et de la protéomique au cours de la dernière décennie ont cependant permis de démontrer que plusieurs gènes impliqués dans la synthèse de métabolites bactériens ne sont pas exprimés dans des conditions de cultures conventionnelles en laboratoire [3].

De nombreuses espèces bactériennes ont la capacité de synthétiser des métabolites pouvant être utilisés comme produits naturels en médecine humaine. Nous savons aussi que cette capacité est sous-exploitée à la lumière des nouvelles connaissances du microbiome et des relations symbiotiques. La co-culture de microorganismes peut s'avérer une stratégie efficace pour activer l'expression de certains gènes responsables de la production d'anti-

microbiens. En effet, certaines voies métaboliques et fonctions cellulaires sont induites uniquement en présence d'autres organismes, souvent dans un but compétitif au sein d'un

$\rightarrow$ Voir la Synthèse de F. Vavre et $P$. Mavingui, $m / s n^{\circ} 11$, novembre 2011, page 95 écosystème donné $[4](\rightarrow)$.

Lorsque ces mêmes microorganismes sont cultivés individuellement, ces voies métaboliques peuvent être réprimées et donc non identifiables. Ces fonctions sont souvent étudiées dans des contextes d'antagonisme, mais peuvent également être retrouvées dans des relations de commensalisme ou de mutualisme. Puisqu'il est actuellement estimé que moins de $1 \%$ des microorganismes présents sur terre ont été cultivés en laboratoire [5], les possibilités de mettre au jour de nouveaux produits naturels antimicrobiens potentiellement utiles en médecine sont innombrables.

La découverte de la teixobactine, un antibiotique à large spectre, a d'ailleurs mis en évidence toute la puissance de la culture «in situ» de microorganismes au sein d'un écosystème complexe [6]. Dans ce cas particulier, grâce à un dispositif multi-puits nommé «iChip », des bactéries du sol ont été incubées en présence d'une communauté complexe de microorganismes au sein de leur habitat naturel (échantillon de sol). Cette culture in situ a permis la croissance de bactéries habituellement «non-cultivables», dont Eleftheria terrae, et ainsi la mise en évidence de la teixobactine comme sous-produit métabolique.
La co-culture bactérienne : ses promesses dans le développement d'antimicrobiens

La capacité de certaines bactéries marines à produire une réponse antagoniste, lorsqu'elles sont exposées à des bactéries terrestres, est particulièrement intéressante dans le cadre du développement de nouveaux agents antimicrobiens. Cette approche est d'ailleurs exploitée depuis la fin des années 1990, quand Burgess et al. ont étudié la réponse de bactéries vivant à la surface d'algues marines mises en présence de bactéries pathogènes, telles que Staphylococcus aureus ou Pseudomonas aeruginosa [7]. La première hypothèse émise alors était que ces bactéries marines avaient la capacité de produire différentes substances antimicrobiennes et que cette production était stimulée par leur interaction avec les bactéries pathogènes. Depuis, d'autres exemples de co-culture ont permis d'identifier de nouveaux antibiotiques potentiels (Tableau I).

$\varepsilon n$ ce sens, Navid Adnani et ses collègues de l'université du Wisconsin ont récemment découvert une nouvelle molécule aux propriétés antimicrobiennes, la keyicine [9]. Cet antibiotique a été découvert par la mise en co-culture de Micromonospora spp., une bactérie associée à des invertébrés marins, et de Rhodococcus, une bactérie terrestre avec un potentiel pathogène. La co-incubation de ces deux bactéries a permis de déclencher la production de la keyicine par la machinerie cellulaire de Micromonospora et ce, malgré l'absence de 


\begin{tabular}{|lll}
\hline Antimicrobien & Producteur & Partenaire \\
\hline Acremostatines A-C & Acremonium sp. & Mycogone rosea \\
\hline Libertellenones A-D & Libertella sp & Q-Proteobactérie marine \\
\hline Glionitrine A & Aspergillus fumigatus & Sphingomonas sp. \\
\hline Secopénicillide C & Penicillium pinophilum & Trichoderma harzianum \\
\hline Analogues de l'acide tétramique & Fusarium pallidoroseum & Saccharopolyspora erythraea \\
\hline Citrifélines A-B & Penicillium citrinum & Beauveria feline \\
\hline Arcyriaflavine $\boldsymbol{\varepsilon}$ & Streptomyces cinnamoneus & Tsukamurella pulmonis \\
\hline Niizalactames A-C & Streptomyces $\mathrm{sp}$. & T. pulmonis \\
\hline Chojalactones A-C & Streptomyces $\mathrm{sp}$. & T. pulmonis \\
\hline
\end{tabular}

Tableau I. Exemples d'antibiotiques en développement et issus d'une méthode de co-culture (adapté de [8]).

contact direct entre les deux espèces bactériennes. Des analyses génomiques et protéomiques ont permis de démontrer que plusieurs gènes de biosynthèse de Micromonospora sont induits par cette technique, mais uniquement en présence de Rhodococcus.

La structure de ce nouvel antibiotique s'apparente aux anthracyclines, une classe d'antimicrobiens principalement utilisée en oncologie pour leurs propriétés antinéoplasiques. La keyicine semble cependant posséder un mécanisme d'action distinct des autres anthracyclines, qui induisent généralement des dommages en s'intercalant dans I'ADN de leur cible.

\section{Potentiel clinique de la keyicine}

Les études préliminaires portant sur la keyicine démontrent une activité sélective contre les bactéries à Gram positif, telles que Staphyloccocus spp. ou Enterococcus spp. Elle est également active contre les mycobactéries et les actinomycètes (comme Rhodococcus spp.). Fait intéressant, cette activité antimicrobienne a été retrouvée autant pour les souches de $S$. aureus sensibles aux antibiotiques de première ligne que pour les souches multirésistantes. Cette activité antimicrobienne sélective est particulièrement prometteuse dans la lutte contre des bactéries résistantes, dans un contexte où, actuellement, près du quart des infections invasives à $S$. aureus observées en France sont résistantes aux antibiotiques de premier choix dans ce type de condition, les bêta-lactamines [10]. La menace de l'Enterococcus faecium résistant à la vancomycine est aussi bien présente, avec environ $5 \%$ des isolats issus de souches invasives. La keycine a donc un potentiel intéressant dans une niche où le nombre d'agents actifs est sans cesse révisé à la baisse.

Bien qu'il ne soit pas complètement élucidé, des données préliminaires suggèrent que le mécanisme d'action de la keyicine n'est pas lié à des dommages causés à I'ADN de la bactérie visée, comme on peut l'observer avec les autres antibiotiques de la famille des anthracyclines qui présentent une importante toxicité chez l'homme. L'interférence des anthracyclines avec l'ADN des cellules myocardiques est, entre autres, responsable d'une cardiotoxicité pouvant mener à une insuffisance cardiaque [11]. Bien que la keyicine n'ait encore jamais été testée chez l'être humain, son développement vers une utilisation clinique plus étendue pourrait donc être favorisée par son mécanisme d'action singulier.

\section{Conclusion}

Le développement de la génomique et de la protéomique ont récemment révélé un nombre inestimable de gènes de biosynthèse dont les produits métaboliques pourraient potentiellement être utiles en clinique, mais qui demeurent silencieux dans des conditions de culture bactériennes conventionnelles. La keyicine est un parfait exemple d'un antibiotique issu de la co-culture entre une bactérie marine associée aux invertébrés et une bactérie terrestre, et son spectre d'activité suggère une utilité potentielle dans la lutte contre les infections causées par des pathogènes à Gram positif multirésistants. Cette découverte nous rappelle aussi l'importance de développer des stratégies de recherche qui s'approchent des conditions de vie réelles des microorganismes afin d'en tirer leur plein potentiel. $\diamond$

Discovery of keyicin, a new antibiotic: it takes two to tango

\section{LIENS D'INTÉRÊT}

Les auteurs déclarent n'avoir aucun lien d'intérêt concernant les données publiées dans cet article.

\section{RÉFÉRENCES}

1. Organisation mondiale de la santé. Plan d'action mondial pour combattre la résistance aux antimicrobiens. www.who.int/antimicrobial resistance/global-action-plan/fr/2016: 1-32. 


\section{RÉFÉRENCES}

2. Katz L, Baltz RH. Natural product discovery: past, present, and future. J Ind Microbiol Biotechnol 2016 ; $43: 155-76$.

3. Cimermancic P, Medema MH, Claesen J, et al. Insights into secondary metabolism from a global analysis of prokaryotic biosynthetic gene clusters. Cell 2014 ; $158: 412-21$.

4. Vavre F, Mavingui P. Les bactéries symbiotiques d'arthropodes et de nématodes. De nouvelles alliées dans le contrôle des maladies infectieuses. Med Sci (Paris) $2011 ; 27: 953-8$.
5. Katz M, Hover BM, Brady SF. Culture-independent discovery of natural products from soil metagenomes. J Ind Microbiol Biotechnol $2015 ; 43$ : 129-41.

6. Ling LL, Schneider T, Peoples AJ, et al. A new antibiotic kills pathogens without detectable resistance. Nature $2015 ; 517: 455$

7. Burgess JG, Jordan EM, Bregu M, et al. Microbial antagonism: a neglected avenue of natural products research. J Biotechnol $1999 ; 70: 27-32$.

8. Ueda K, Beppu T. Antibiotics in microbial coculture.J Antibiot (Tokyo) $2017 ; 70: 361-5$.

9. Adnani N, Chevrette MG, Adibhatla SN, et al. Coculture of marine invertebrate-associated bacteria and

\section{NOUVELL}

Des approches multi-omiques
dévoilent de nouvelles
cibles thérapeutiques
pour le traitement
du cancer du pancréas

Rémy Nicolle ${ }^{1}$, Yuna Blum ${ }^{1}$, Laetitia Marisa ${ }^{1}$, Nelson Dusetti ${ }^{2}$, Juan lovanna ${ }^{2}$

Les faux espoirs de la génomique de l'adénocarcinome pancréatique fondés sur les mutations génétiques Au cours des dernières décennies, l'analyse génétique détaillée des tumeurs a permis l'identification et la validation de gènes mutés et fréquemment dérégulés dans les tumeurs, indiquant une dépendance génétique de leur développement. Les données recueillies ont ainsi suggéré qu'il serait possible de tirer parti de ces mutations pour un ciblage thérapeutique lorsque des médicaments spécifiques étaient disponibles. Après une période très enthousiaste, il s'avère que ces cibles ne peuvent, en fait, être exploitées que chez un faible pourcentage de patients, soit parce que les médicaments pertinents ne sont pas disponibles, soit parce que les gènes mutés ne sont pas accessibles par des approches pharmacologiques. Les mutations de gènes observées dans les adénocarcinomes pancréatiques sont relativement conservées entre les tumeurs (KRAS, P53, SMAD4, CDKN2A, MLL3, TGFBR2,
ARIDIA et $S F 3 B I)^{1}$, mais les gènes susceptibles d'être ciblés restent extrêmement rares. La majorité d'entre eux ne sont en effet pas associés à une activité enzymatique ou, lorsqu'elle existe, elle est souvent fondée sur une interaction protéine-protéine qui reste techniquement difficile à déstabiliser.

L’adénocarcinome pancréatique, une maladie extrêmement hétérogène L'adénocarcinome pancréatique (AP) est l'une des affections malignes les plus meurtrières. II est la cause d'environ 350000 décès par an dans le monde [1]. Le pronostic de cette maladie est très mauvais avec environ seulement $5 \%$ des patients ayant une survie supérieure à 5 ans après le diagnostic [1]. Les nombreux essais cliniques n'ont pas

\footnotetext{
${ }^{1}$ Ces mutations affectent les gènes : KRAS (Kirsten rat sarcoma virus), P53, SMAD4 (mothers against decapentaplegic homolog 4), CDKN2A (cyclin-dependent kinase 2a/p16), MLL3 (lysine methyltransferase 2C), TGF $\beta$ R2 (transforming growth factor, beta receptor II), ARIDIA (AT rich interactive domain IA [SWI-like]) et SF3BI (splicing factor $3 b$ subunit 1).
}

interdisciplinary technologies enable biosynthesis and discovery of a new antibiotic, Keyicin. ACS Chem Biol 2017 ; 12 : 3093-102.

10. European centre for disease prevention and control. surveillance of antimicrobial resistance in Europe 2016. https://ecdc.europa.eu/.../antimicrobialresistance-surveillance-europe-2016 $2017: 1-100$.

11. Levis BE, Binkley PF, Shapiro CL. Cardiotoxic effects of anthracycline-based therapy: what is the evidence and what are the potential harms? Lancet Oncol 2017 ; 18 : e445-56.

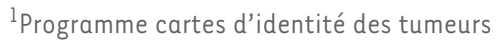 \\ (CIT), Ligue nationale contre le cancer, \\ 14, rue Corvisart, 75013 Paris, France. \\ ${ }^{2}$ Centre de recherche en cancérologie \\ de Marseille (CRCM), Inserm U1068, \\ CNRS UMR 7258, Institut Paoli-Calmettes, \\ Aix Marseille université, \\ 163, avenue de Luminy, 13288 Marseille, \\ France. \\ juan.iovanna@inserm.fr
}

démontré de bénéfices significatifs en termes de survie, probablement parce que les populations de patients incluses dans ces études sont très hétérogènes. Cette hétérogénéité est illustrée par l'évolution clinique que présentent les patients, avec un temps de survie après le diagnostic variant de 2-3 mois à plus de 5 ans, ainsi qu'une forte différence de sensibilité aux traitements. Par exemple, on observe un taux de réponse objective de $31,6 \%$ chez les patients traités par le folfirinox et de 9,4\% chez ceux traités par la gemcitabine, ce qui correspond respectivement à environ $70 \%$ et $90 \%$ de non-répondeurs à ces traitements $[2,3]$. Cette hétérogénéité tumorale s'explique également par le fait que chaque AP est contrôlé par la combinaison de plusieurs voies intracellulaires différentes, ce qui entraîne une susceptibilité variable aux médicaments, au développement de métastases, et donc à la survie [4-6]. Par manque de connaissance de l'importance de cette hétérogénéité de réponses aux traitements, les protocoles cliniques 